\title{
Laboratory production of magnesium sulfide grains and their characteristic infrared spectra due to shape
}

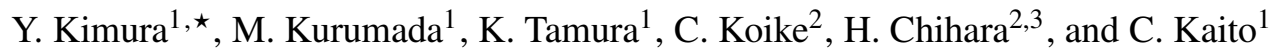 \\ ${ }^{1}$ Department of Physics, Ritsumeikan University, Kusatsu-shi, Shiga 525-8577, Japan \\ e-mail: rp092955@se.ritsumei.ac.jp \\ 2 Laboratory of Physics, Kyoto Pharmaceutical University, Misasagi, Yamashina, Kyoto 607-8414, Japan \\ 3 Department of Earth and Space Science, Osaka University, 1-1, Machikaneyama, Toyonaka, Osaka 560-0043, Japan
}

Received 24 January 2005 / Accepted 13 May 2005

\section{ABSTRACT}

Nanosized MgS grains, which have been considered the origin of the $30 \mu \mathrm{m}$ emission feature of carbon-rich evolved objects, were produced from the gas phase using an advanced gas evaporation method. The far-infrared spectrum of cubic $\mathrm{MgS}$ grains showed a characteristic absorption peak at $311 \mathrm{~cm}^{-1}(32.1 \mu \mathrm{m})$ with three shoulders at 460, 400 and $262 \mathrm{~cm}^{-1}(21.7,25.0$ and $38.2 \mu \mathrm{m})$. On the other hand, when the grains were roundish or network-like, the absorption peak at $250 \mathrm{~cm}^{-1}$ became predominant. The cubic $\mathrm{MgS}$ grains were produced by direct nucleation from the gas phase. In the case of production via a gas-solid reaction, the $\mathrm{MgS}$ grains were network-like. Therefore, the formation environments of $\mathrm{MgS}$ grains around carbon-rich evolved objects may be predicted from the intensity of 310 and $250 \mathrm{~cm}^{-1}$ bands. We suggest that the origins of the absorption band at 310 and $250 \mathrm{~cm}^{-1}$ are (100), (110) and/or (111) surfaces of MgS grains, respectively.

Key words. methods: laboratory - stars: AGB and post-AGB - infrared: ISM - infrared: stars

\section{Introduction}

Infrared observations of carbon stars and some planetary nebulae (e.g., Forrest et al. 1981) have revealed a strong emission feature beginning from $24 \mu \mathrm{m}$ and extending beyond $30 \mu \mathrm{m}$. Condensation calculations for carbon-rich systems (Lattimer et al. 1978) predicted the formation of iron carbide and various metal sulfides, such as $\mathrm{MgS}$ and $\mathrm{FeS}$, as the gas cools. Later, it was simultaneously reported that the infrared spectra of metallic sulfides were measured in the laboratory for commercial materials (Nuth et al. 1985) and the first identification of the $30 \mu \mathrm{m}$ band with $\mathrm{MgS}$ was based on the laboratory measurements by Nuth (Goebel \& Moseley 1985). Recently, contribution of oxygen atoms in the form of hydroxyl groups $(\mathrm{OH})$ in carbonaceous materials has been reported as a candidate source of the $30 \mu \mathrm{m}$ feature (Papoular 2000). Then, in addition to the $30 \mu \mathrm{m}$ feature, the oxygen atoms also contribute to 20 and $26 \mu \mathrm{m}$ features, which are simultaneously seen at stellar evolutionary stages between the end of the asymptotic giant blanch and the planetary nebulae.

Important factors in the determination of spectral features are the size, shape and structure of grains. In particular, it is known that the absorption cross section of $\mathrm{MgS}$

* Present address: Astrochemistry Laboratory, Code 691, Solar System Exploration Division, NASAfs Goddard Space Flight Center, Greenbelt, MD 20771, USA. and $\mathrm{MgO}$ around $30 \mu \mathrm{m}$ is very sensitive to grain shape. Begemann et al. (1994) have confirmed the identification of the $30 \mu \mathrm{m}$ band based on new optical constants of $\mathrm{Mg}_{x} \mathrm{Fe}_{(1-x)} \mathrm{S}$. The optical constants have given reasonable fits for several sources (Jiang et al. 1999; Szczerba 1999). Absorption cross sections for various grain shapes and shape distributions in the Rayleigh limit were calculated in more detail using the refractive index of $\mathrm{Mg}_{0.9} \mathrm{Fe}_{0.1} \mathrm{~S}$ by Begemann et al. (1994) (Hony et al. 2002). When the shape was altered from spherical to ellipsoidal, the feature broadens and the peak position shifts to longer wavelengths from $\sim 28$ to $\sim 39 \mu \mathrm{m}$. Similarly, peak shifts based on temperature effects were also calculated. However, since experimental studies in laboratory are lacking, the correlation between the morphology of $\mathrm{MgS}$ grains and their infrared spectra has yet to be demonstrated. The shape alteration of grains with the same structure is very difficult to determine in a laboratory approach. A shift of an absorption peak of cubic and spherical $\mathrm{MgO}$ grains has been reported, which was coincident with the theoretical calculation by Bohren \& Huffman (1983) (Kimura et al. 1997). We have systematically studied the correlation between the infrared spectra, size and structure of TiC grains using a transmission electron microscope (TEM) in the nanoregion (Kimura \& Kaito 2003; Kimura et al. 2004). The spectrum of TiC nanoparticles is influenced by slight structural changes and the presence of defects in the 

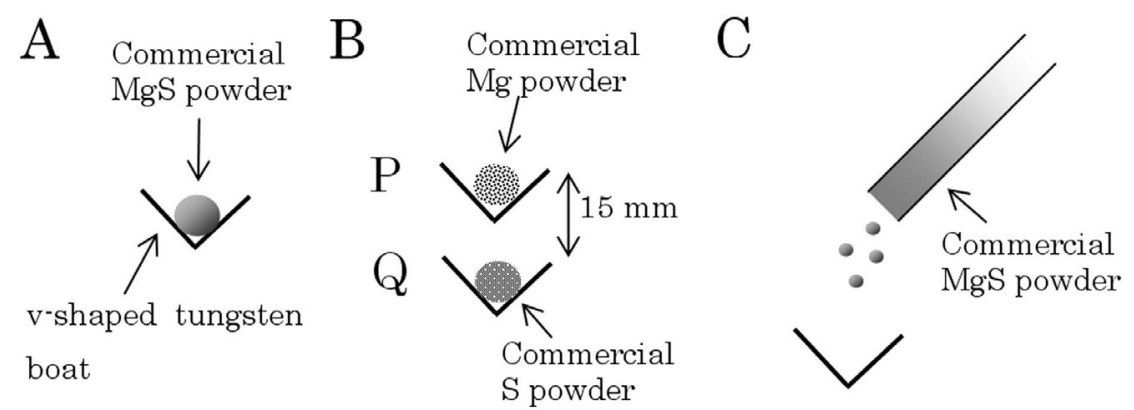

Fig. 1. Schematic representations of production methods of MgS grains: A, typical gas evaporation method; B, two boats method; C, flash evaporation method.

nanoregion. In the present paper, we will investigate spectral changes for $\mathrm{MgS}$ grains condensed from the gas phase. We demonstrate that these infrared spectral features showed a significant dependence on the shape and/or surface of grains.

\section{Experimental methods}

In the present experiment, three gas evaporation methods were attempted, as shown in Fig. 1. Method A is a typical gas evaporation method (Kaito 1978), and Methods B and C are advanced gas evaporation methods called the two boats method (Kaito et al. 1989) and the flash evaporation method (Tanigaki et al. 2002), respectively. The details will be described in Sect. 3. The evaporation chamber was a glass cylinder $17 \mathrm{~cm}$ in diameter and $30 \mathrm{~cm}$ in height, covered with a stainless-steel plate on top and connected to a high-vacuum exhaust through a valve at the bottom. After $\mathrm{MgS}$ or $\mathrm{Mg}$ and $\mathrm{S}$ commercial powders were placed on the v-shaped tungsten boats, as indicated in Fig. 1, the chamber was evacuated at $10^{-3} \mathrm{~Pa}$. Ar gas was then introduced at $10 \mathrm{kPa}$. The samples were evaporated by heating the boats. During heating, smoke rising from the evaporation source can be observed. The vapor subsequently cools and condenses in the gas atmosphere, and solid grains are obtained directly from the gas cloud. The motion of grains follows convection currents produced by heating, and looks like that of smoke. These smoke grains were collected on a glass funnel $10 \mathrm{~cm}$ above the evaporation source.

These collected specimens were mounted on an amorphous carbon film supported by a standard TEM grid. The specimens were observed using a Hitachi H-7100R TEM equipped with an energy-dispersion X-ray (EDX) analysis system (Horiba Xerophy), and also using a Hitachi H-9000NAR highresolution TEM (HRTEM). The far-infrared spectra, ranging from 650 to $50 \mathrm{~cm}^{-1}(15.4-200 \mu \mathrm{m})$, of samples embedded in polyethylene pellets at a concentration of less than $1 \%$ were measured with a Nicolet Nexus 670 FT-IR spectrometer. The wavelength resolution was $0.5 \mathrm{~cm}^{-1}$. This mixture was then heated to $140{ }^{\circ} \mathrm{C}$ in air to melt polyethylene. To confirm the alteration of samples due to heating in air, the infrared spectra of the same specimens sandwiched between polyethylene sheets were also observed. As a result, the influences of heating in air on the spectral feature were found to be negligible throughout the present experiment.

\section{Procedures and products of each method}

In the case of method A, a typical gas evaporation method, $\mathrm{MgS}$ commercial powder, which was placed onto the v-shaped tungsten boat, was evaporated by heating the boat in Ar gas, as shown in Fig. 1A. As a result of a TEM analysis of collected grains, $\mathrm{MgS}$ grains were produced in small amounts, although sulfur atoms were sufficiently obtained as shown by the EDX spectrum. Since the temperature of the boat was gradually increased during the heating, $\mathrm{MgS}$ commercial powder was decomposed before its evaporation. As a result, $\mathrm{Mg}$ and $\mathrm{S}$ are evaporated individually. Therefore, $\mathrm{MgS}$ grains were not the main product.

In the case of method $\mathrm{B}$, the two boats method, $\mathrm{Mg}$ and $\mathrm{S}$ commercial powders were placed onto the v-shaped tungsten boats $\mathrm{P}$ and $\mathrm{Q}$, respectively. Boat $\mathrm{Q}$ was set $15 \mathrm{~mm}$ below boat $\mathrm{P}$. By controlling the heating temperature of boats $\mathrm{P}$ and $\mathrm{Q}$ at about 600 and $350^{\circ} \mathrm{C}$, respectively, $\mathrm{Mg}$ and $\mathrm{S}$ simultaneously evaporated at a vapor pressure of $100 \mathrm{~Pa}$ order. The evaporated $\mathrm{S}$ powder rose above boat $\mathrm{P}$ by riding the convection flow produced by the heating of the two boats. As a result, $\mathrm{MgS}$ grains were produced at the confluence around boat $\mathrm{P}$. The collected grains were composed of $\mathrm{Mg}, \mathrm{MgO}$ crystallites, crystalline MgS and amorphous MgS. Using the two boats method, the control of grain production was difficult throughout the experiments, i.e., $\mathrm{MgS}$ grains were not the main product.

In the case of method $\mathrm{C}$, the flash evaporation method, $\mathrm{MgS}$ commercial powder was dropped into the heated boat, as shown in Fig. 1C. The MgS commercial powder evaporates before it comes into contact with the heated boat. Therefore, even when $\mathrm{MgS}$ was decomposed, the evaporated amounts of $\mathrm{Mg}$ and $S$ were maintained throughout the experiment. Therefore, $\mathrm{MgS}$ grains could be mostly produced. Using the flash evaporation method nanosized grains of an evaporant sample are easily obtained.

\section{TEM observation and infrared spectra of produced MgS grains}

Figure 2 shows a TEM image and the corresponding ED pattern of commercial $\mathrm{MgS}$ powder (Kojundo Chemical Lab. Co., Ltd., Japan, 99.9\%). The commercial MgS powder was roundish cubic and the size was $50-150 \mathrm{~nm}$, which was assembled as seen in the TEM image of Fig. 2. In addition to the ED rings of $\mathrm{MgS}$, 


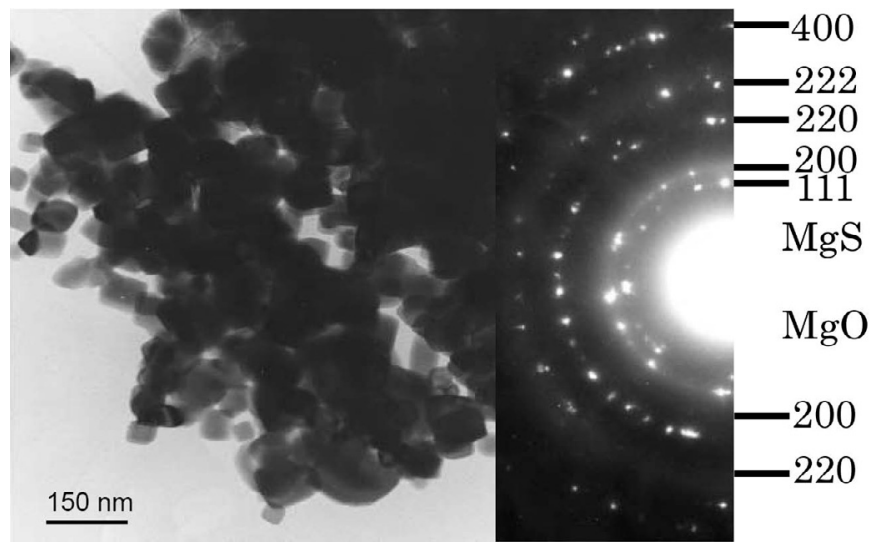

Fig. 2. Typical TEM image and corresponding ED pattern of commercial MgS powder (Kojundo Chemical Lab. Co., Ltd., Japan, 99.9\%).

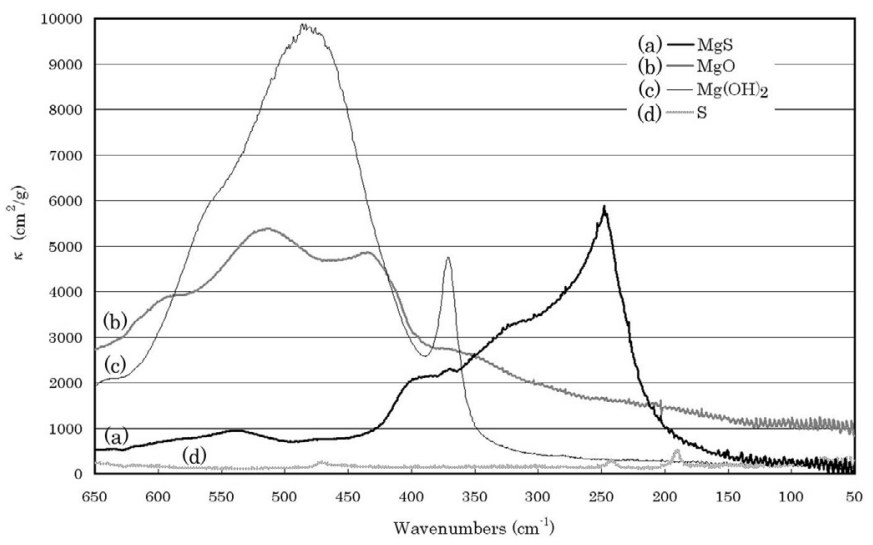

Fig. 3. Infrared spectra of commercial powder ranging from 650 to $50 \mathrm{~cm}^{-1}$. a): corresponding to commercial $\mathrm{MgS}$ powder as shown in Fig. 2; b): commercial $\mathrm{MgO}$ powder; c): commercial $\mathrm{Mg}(\mathrm{OH})_{2}$ powder. d) commercial $\mathrm{S}$ powder.

two broad ED rings corresponding to (200) and (220) of $\mathrm{MgO}$ can be also observed. The size of $\mathrm{MgO}$ crystallites was approximately $1 \mathrm{~nm}$, which was estimated from the diffuseness of the diffraction ring. Since $\mathrm{MgS}$ oxidizes in air, the measurement of reliable data of pure $\mathrm{MgS}$ is difficult (Begemann et al. 1994). Note that the TEM observation and measurement of the infrared spectrum were carried out immediately after the purchase of the commercial MgS powder. The infrared spectrum of the commercial $\mathrm{MgS}$ powder showed the characteristic features, which are composed of a markedly strong peak at $248 \mathrm{~cm}^{-1}(40.3 \mu \mathrm{m})$ with three shoulders at 324,370 and $400 \mathrm{~cm}^{-1}(30.9,27.0$ and $25.0 \mu \mathrm{m})$ and a small bump at $538 \mathrm{~cm}^{-1}(18.6 \mu \mathrm{m})$ within our measurement limit $(650$ to $\left.50 \mathrm{~cm}^{-1}(15.4-200 \mu \mathrm{m})\right)$, as indicated in Fig. 3a. The infrared spectra of commercial $\mathrm{MgO}, \mathrm{Mg}(\mathrm{OH})_{2}$ and $\mathrm{S}$ powders are also displayed in Figs. 3b-d for the comparison to $\mathrm{MgS}$ spectra. The small bump at $538 \mathrm{~cm}^{-1}(18.6 \mu \mathrm{m})$ of the spectrum of commercial $\mathrm{MgS}$ powder may be attributed to the surface oxide ( $\mathrm{MgO}$ crystallites) of $\mathrm{MgS}$ powder.

Figures $4 \mathrm{a}$ and $4 \mathrm{~b}$ show the typical TEM images and the corresponding ED patterns of grains produced by method $\mathrm{A}$ and $\mathrm{B}$, respectively. These TEM observations were immediately carried out within 15 min after the production of grains.

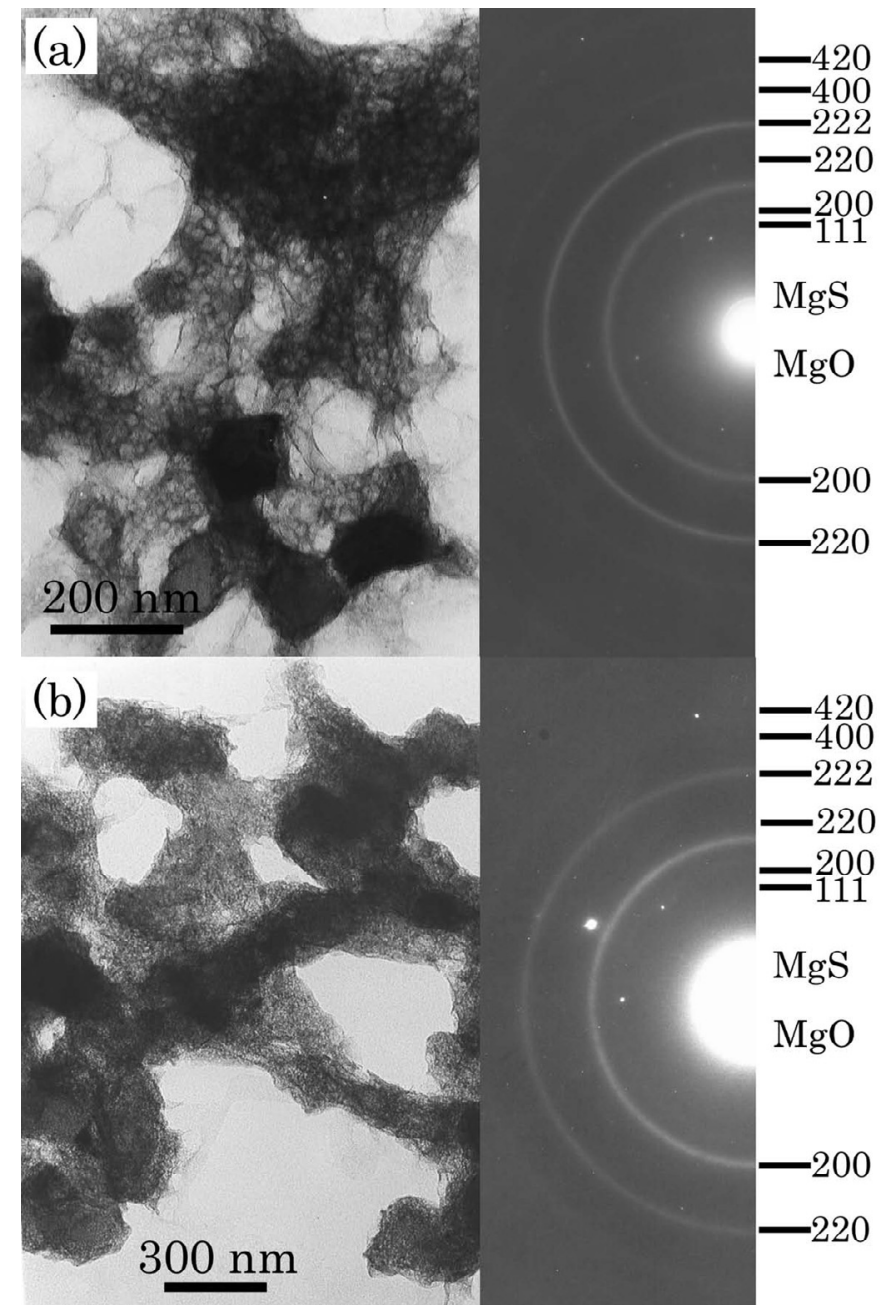

Fig. 4. a) and b) show the typical TEM images and ED patterns of the collected $\mathrm{MgS}$ grains produced by methods $\mathrm{A}$ and $\mathrm{B}$, respectively. Many nanocrystallites of $\mathrm{MgO}$ were produced in both types of specimen, since diffuse ED rings attributed to $\mathrm{MgO}$ are visible.

The grains produced by both methods did not shown the characteristic crystal habit of $\mathrm{NaCl}$-type structure. The shape is network-like. Many voids, which are visible as white spherical contrasts, are formed in the grains produced by both methods. Generally, these voids are generated by reactions on grain surfaces, such as the oxidation of metallic particles (Kaito et al. 1973). Therefore, both types of specimen will be produced via the solid-vapor reaction, i.e., the reaction between $\mathrm{Mg}$ solid and $S$ vapor. Their infrared spectra are shown in Figs. 5a and 5b, respectively. The infrared spectrum of commercial MgS powder is also displayed in Fig. 5d. The spectrum in Fig. 5a exhibits the characteristic three peaks at 376,427 and $580 \mathrm{~cm}^{-1}(26.6,23.4$ and $17.2 \mu \mathrm{m}$ ), which are mainly attributed to $\mathrm{MgO}$. It is well coincident with the strongly diffused rings in the ED pattern, indicating that the specimen is composed of MgO nanocrystallites. On the other hand, the spectrum in Fig. 5b exhibits peaks at 250 and $309 \mathrm{~cm}^{-1}$ (40.0 and $32.4 \mu \mathrm{m}$ ) attributed to $\mathrm{MgS}$ in addition to the two peaks at 402 and $580 \mathrm{~cm}^{-1}(24.9$ and $17.2 \mu \mathrm{m}$ ) attributed to $\mathrm{MgO}$. 


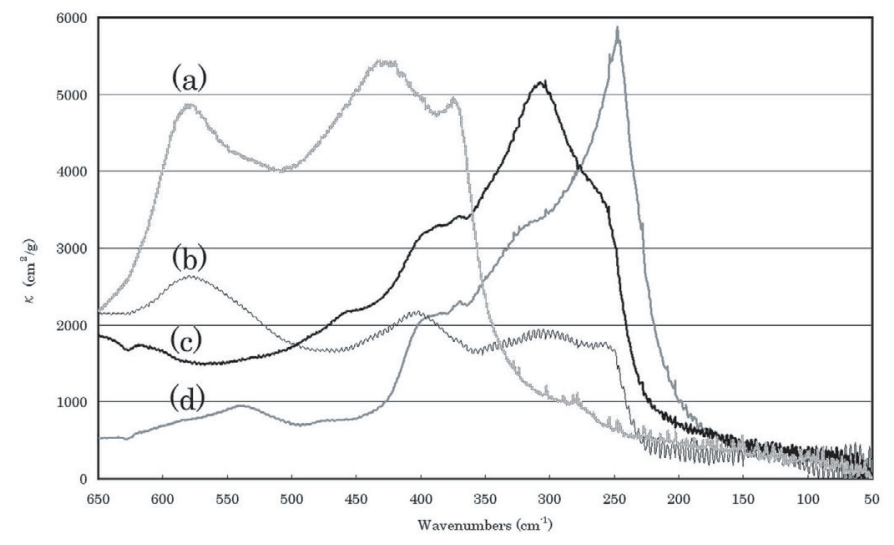

Fig. 5. Infrared spectra of produced $\mathrm{MgS}$ grains ranging from 650 to $50 \mathrm{~cm}^{-1}$ are shown in a)-c). a) corresponding to $\mathrm{MgS}$ grains as shown in Fig. 4a which were produced by method A. The characteristic three peaks are seen at 376,427 and $580 \mathrm{~cm}^{-1}(26.6,23.4$ and $17.2 \mu \mathrm{m}$ ). b) corresponding to $\mathrm{MgS}$ grains as shown in Fig. $4 \mathrm{~b}$, which were produced by method B. The characteristic four peaks are seen at 250,309, 402 and $580 \mathrm{~cm}^{-1}(40.0,32.4,24.9$ and $17.2 \mu \mathrm{m})$. c) corresponding to $\mathrm{MgS}$ grains as shown in Fig. 6 which were produced by method $\mathrm{C}$. The characteristic four peaks are seen at 460, 400, 311 and $262 \mathrm{~cm}^{-1}(21.7,25.0,32.1$ and $38.2 \mu \mathrm{m})$. The infrared spectrum corresponding to the commercial $\mathrm{MgS}$ powder is also indicated in d).

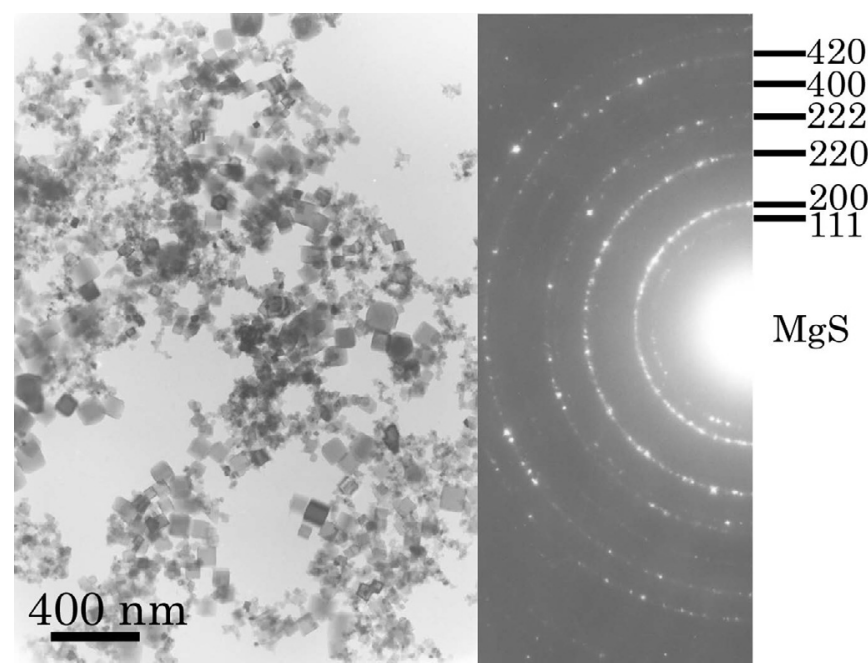

Fig. 6. Typical TEM image and ED pattern of collected MgS grains produced by method $\mathrm{C}$. The ED pattern clearly shows the formation of $\mathrm{MgS}$ grains.

The TEM image and corresponding ED pattern of typical grains produced by method $\mathrm{C}$ are shown in Fig. 6 . The ED pattern clearly shows the production of $\mathrm{MgS}$ grains. The grains are $10-100 \mathrm{~nm}$ in diameter and the majority are $10-30 \mathrm{~nm}$ in size. The produced $\mathrm{MgS}$ grains were typically cubic indicating the NaCl-type structure. In contrast to the commercial $\mathrm{MgS}$ powder, ED rings corresponding to $\mathrm{MgO}$ were rarely observed. A HRTEM image of typical $\mathrm{MgS}$ grains approximately $35 \mathrm{~nm}$ in size is shown in Fig. 7. The lattice planes of $0.260 \mathrm{~nm}$ corresponding to (200) of MgS are clearly observed through a grain. Although the ED pattern of $\mathrm{MgO}$ was not detected, $\mathrm{MgO}$ nanocrystallites less than $5 \mathrm{~nm}$ in size are observed on the surfaces of $\mathrm{MgS}$ grains, as indicated by circles. Moiré fringes in $\mathrm{MgS}$ grains, as indicated by dotted circles in Fig. 7, correspond to interference between the (200) lattice planes of $\mathrm{MgS}$ grain and $\mathrm{MgO}$ surface crystallites.

The infrared spectrum of these $\mathrm{MgS}$ grains is shown in Fig. 5c. Four characteristic peaks can be seen at 460, 400, 311 and $262 \mathrm{~cm}^{-1}(21.7,25.0,32.1$ and $38.2 \mu \mathrm{m})$. The remarkable difference between the spectrum of the commercial MgS powder shown in Fig. 5d and that of produced $\mathrm{MgS}$ grains shown in Fig. $5 \mathrm{c}$ is in the intensity of 310 and $250 \mathrm{~cm}^{-1}$ bands. The $310 \mathrm{~cm}^{-1}$ band of the produced MgS grains became significantly more intense in contrast to the $250 \mathrm{~cm}^{-1}$ band, which dominates in the spectrum shown in Fig. $5 \mathrm{~d}$. The origins of these two bands at 310 and $250 \mathrm{~cm}^{-1}$ can be attributed to the shape and/or surface of the grains. Generally, peak shifts are demonstrated by the grain shape. It was calculated that for spherical $\mathrm{MgS}$ grains the peak is shifted from $\sim 370 \mathrm{~cm}^{-1}$ $(\sim 27 \mu \mathrm{m})$ to $\sim 260 \mathrm{~cm}^{-1}(\sim 38 \mu \mathrm{m})$ for needle-shaped grains (Hony et al. 2002). It has also been calculated that the main resonances of cubic $\mathrm{MgO}$ particles are between those of spherical and needle-shaped particles (Bohren \& Huffman 1983). Since $\mathrm{MgS}$ is of the same structure as $\mathrm{MgO}$, the character of the absorption should be similar, i.e., the absorption of cubic $\mathrm{MgS}$ grains will be located between those of spherical and needle-shaped grains in the calculation. Indeed, in the present study, cubic $\mathrm{MgS}$ grains show the absorption peak at $311 \mathrm{~cm}^{-1}(32.1 \mu \mathrm{m})$ which is between $\sim 370 \mathrm{~cm}^{-1}$ of spherical and $\sim 260 \mathrm{~cm}^{-1}$ of needle-shaped grains, as shown in Fig. 5c. However, roundish cubic $\mathrm{MgS}$ grains show a main peak at $248 \mathrm{~cm}^{-1}$ which is different from the $\sim 370 \mathrm{~cm}^{-1}$ of the calculation by Hony et al. (2002). Therefore, we will suggest other possibilities regarding the origin of the two peaks.

In our previous study, we demonstrated using nanosized TiC grains with different geometries and sizes that the absorption features at 12.5 and $14.3 \mu \mathrm{m}$ originate from the surface rather than the grain size, i.e., the (100) surface of carbonrich $\mathrm{TiC}$ grains and (111) surface composed of $\mathrm{Ti}$ atoms, respectively (Kimura et al. 2004). In this study, the surface of roundish cubic commercial $\mathrm{MgS}$ powder is surrounded by (111) and (110) in addition to (100). On the other hand, the surface of produced cubic $\mathrm{MgS}$ grains is mostly surrounded by (100). Therefore, it can be concluded that the origin of $310 \mathrm{~cm}^{-1}$ is (100) and that of $250 \mathrm{~cm}^{-1}$ is (110) and/or (111). This hypothesis is coincident with the spectrum shown in Fig. 5b. The spectrum (Fig. 5b) from a network-like grain shown in Fig. $4 \mathrm{~b}$ with various surfaces including (100), (110) and (111) shows similar intensity between two peaks at 310 and $250 \mathrm{~cm}^{-1}$ which hardly shifted in position.

Another possibility explaining the strong $250 \mathrm{~cm}^{-1}$ from roundish cubic $\mathrm{MgS}$ grains can be considerable the coagulation of the grains. Although it has been reported that the infrared spectrum of particles is affected by the agglomeration (Clément et al. 2003), the correlation between the state of agglomeration and spectral changes is not sufficiently known. The agglomeration effects have not been seen or noticed in the nanoparticles produced by the gas-evaporation methods. However, in the case of the commercial MgS particles, the particles are heavily agglomerated and coalesced compared with 


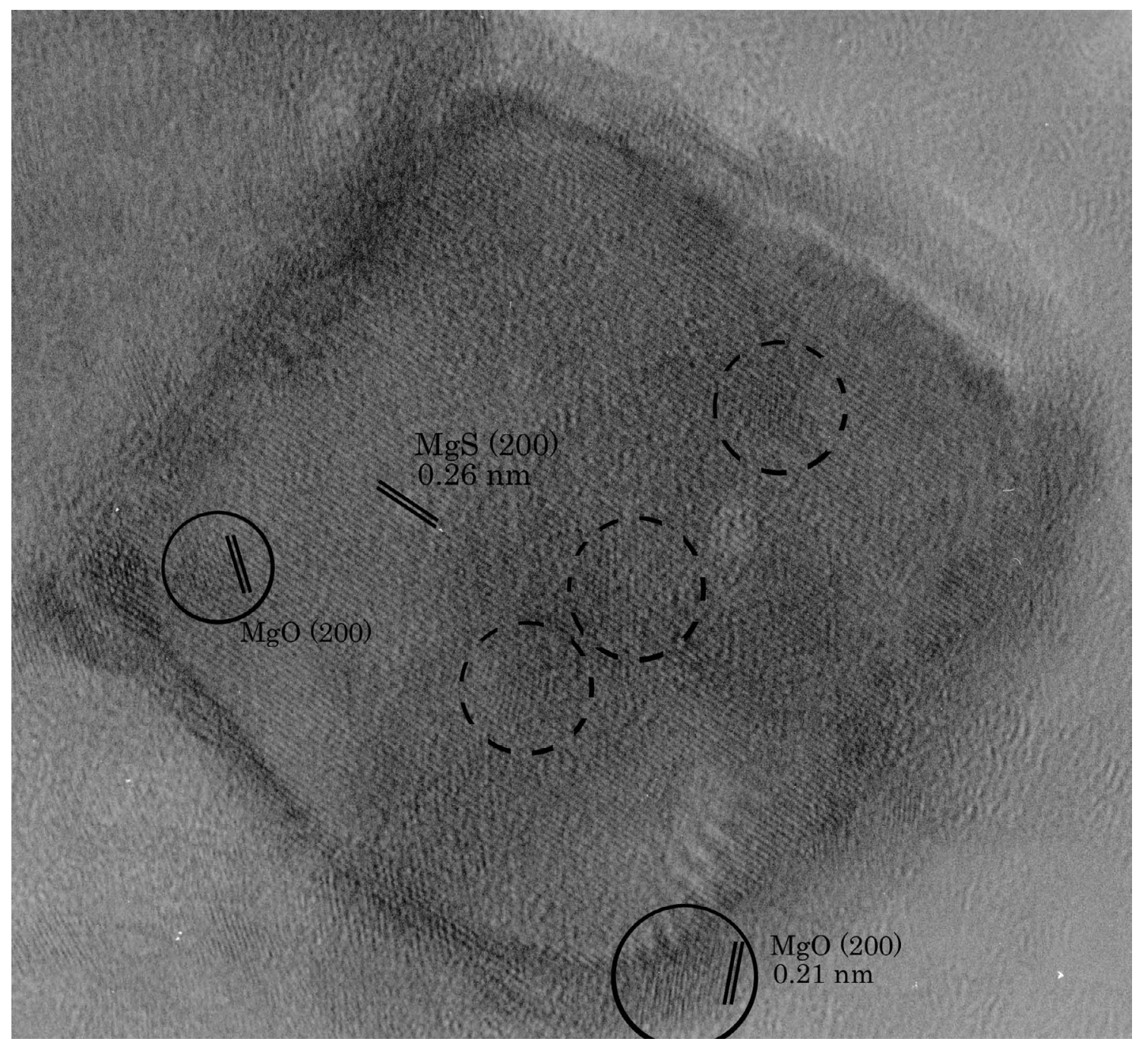

Fig. 7. Typical HRTEM image of produced $\mathrm{MgS}$ grains. The formation of $\mathrm{MgO}$ crystallites can be observed as indicated by circles, although it was observed immediately after $\mathrm{MgS}$ grain production. Dotted circles indicate the Moiré fringes corresponding to the correlation between the (200) lattice planes of $\mathrm{MgS}$ grains and surface $\mathrm{MgO}$ crystallites.

gas-evaporated particles. Accordingly, the agglomeration effect may not be negligible. Here, the agglomeration effect can be considered a surface effect. When the coalescence among the $\mathrm{MgS}$ grains occurs, (100) surfaces are predominantly coalesced due to their $\mathrm{NaCl}$-type structure. As a result, the absorption intensity at $310 \mathrm{~cm}^{-1}$ is more suppressed by the decrease of (100) surfaces. Therefore, it can be concluded that the absorption intensity at $250 \mathrm{~cm}^{-1}$ becomes strong in comparison with $310 \mathrm{~cm}^{-1}$. We suggest that the surface effect is more plausible than the geometrical effect, i.e., cubic or round shape as the origin of the 310 and $250 \mathrm{~cm}^{-1}$ bands in the nanoregion.

\section{Summary}

Although the measurement of completely oxideless $\mathrm{MgS}$ grains could not be carried out, we demonstrated that the spectrum of $\mathrm{MgS}$ grains produced from the gas phase shows the characteristic feature at $311 \mathrm{~cm}^{-1}(32.1 \mu \mathrm{m})$. When $\mathrm{MgS}$ grains are produced directly from the gas phase in the ejected gas from objects, the grains become cubic. The infrared spectrum then shows the $310 \mathrm{~cm}^{-1}$ band, although it should depend on temperature. On the other hand, when $\mathrm{MgS}$ grains are produced via a gas-solid reaction, the $250 \mathrm{~cm}^{-1}$ band becomes more intense than the $310 \mathrm{~cm}^{-1}$ band. We conclude that, according to the band profile, it can be decided whether grains in cosmic environments have formed via direct gas-phase condensation or a gas-solid reaction.

Acknowledgements. This work was partially supported by grants from JSPS Research Fellowships for Young Scientists from April 2002 to March 2004 and Postdoctoral Fellowships for Research Abroad from April 2004 to March 2006.

\section{References}

Begemann, B., Dorschner, J., Henning, T., Mutschke, H., \& Thamm, E. 1994, ApJ, 423, L71

Bohren, C. F., \& Huffman, D. R. 1983, Absorption and Scattering of Right by Small Particles (Wiley Interscience)

Clément, D., Mutschke, H., Klein, R., \& Henning, Th. 2003, ApJ, 594, 642 
Forrest, W. J., Houck, J. R., \& McCarthy, J. F. 1981, ApJ, 248, 195

Goebel, J. H., \& Moseley, H. 1985, ApJ, 290, L35

Hony, S., Waters, L. B. F. M., \& Tielens, A. G. G. M. 2002, A\&A, 390, 533

Jiang, B. W., Szczerba, R., \& Deguchi, S. 1999, A\&A, 344, 918

Kaito, C., Fujita, K., \& Hashimoto, H. 1973, Jpn J. Appl. Phys., 12, 489

Kaito, C. 1978, Jpn. J. Appl. Phys., 17, 601

Kaito, C., Saito, Y., \& Fujita, K. J. 1989, J. Crystal Growth, 94, 967

Kimura, S., Kaito, C., Tamura, N., Saito, Y., \& Koike, C. 1997, Antarctic Meteorites XXII (Tokyo: National Institute of polar research), 85
Kimura, Y., \& Kaito, C. 2003, MNRAS, 343, 385

Kimura, Y., Ikegami, A., Kurumada, M., Kamitsuji, K., \& Kaito, C. 2004, ApJS, 152, 297

Lattimer, J. M., Schramm, D. N., \& Grossman, L. 1978, ApJ, 219, 230

Nuth, J. A., Moseley, S. H., Silverberg, R. F., Goebel, J. H., \& Moore, W. J. 1985, ApJ, 290, L41

Papoular, R. 2000, A\&A, 362, L9

Szczerba, R., Henning, T., Volk, K., Kwok, S., \& Cox, P. 1999, A\&A, 345, L39

Tanigaki, T., Kimura, S., Tamura, N., \& Kaito, C. 2002, Jpn J. Appl. Phys., 41, 5529 